\title{
The Sorites Paradox, "Life," and Abiogenesis
}

\author{
Barend Vlaardingerbroek
}

Published online: 31 March 2012

(C) Springer Science+Business Media, LLC 2012

\begin{abstract}
The ancient Greek philosopher Eubulides of Miletus drew attention to the impossibility of defining a point of transition between two states or conditions at opposite ends of a continuum. The ensuing "drawing the line" conundrumthe sorites paradox - arises from the vague predicates which humans use to convey concepts. It is argued that "life" is an indeterminate concept subject to the vagaries of the paradox. Science communicators and educators need to refrain from phraseology which connotes a sharp line of demarcation between nonliving and living or the existence of a discrete moment in time at which life appeared.
\end{abstract}

Keywords Abiogenesis $\cdot$ Sorites paradox $\cdot$ Definition (of life) - Predicates

\section{The Sorites Paradox}

Eubulides of Miletus was a contemporary of Aristotle in the fourth century BCE who was famous for his paradoxes, the best known being the "liar paradox" ("I am a telling a lie," which, if either a true or a false statement, leads to the negation of the principal assertion). Less well known but the subject of considerable reappraisal by philosophers over recent years is the "sorites paradox": beginning with a pile of stones and removing these one stone at a time, one moves from a pile to a non-pile, but it is impossible to specify the individual stone; the removal of which effects the transition. Moreover, if no one stone can be identified which separates the "pile" from the "not a pile" state, then neither can the stone on either side

B. Vlaardingerbroek $(\bowtie)$

American University of Beirut,

Beirut, Lebanon

e-mail: bv00@aub.edu.lb thereof (or two, three or more stones removed) be designated as being so. Another classical example of the paradox is the transition from baldness to hirsuteness: no given strand of hair, when moving from a bald head to a hirsute one, can be identified as bringing about the change of condition. And yet the difference between a pile of stones and a handful of stones, or a bald head and a luxuriant one, is readily observable.

The sorites paradox draws attention to the impossibility of defining the precise moment of transition from one state to another when there is a continuum between the two. In semantic terms, the paradox applies to concepts with vague predicates (Hyde 2011). Terms such as "pile" and "hirsute" do not have clear boundaries. They may be envisaged as convenient descriptors of phenomena in overtly recognizable form, but cannot be precisely delineated. Words being conveyors of concepts, the spotlight then shifts to the preciseness of human concepts. The enlightenment and logical positivism ushered in a period when it was thought that the world could be described in Euclidean terms, i.e., one of pat, precise definitions (Hogan 1994). But the real world does not conform very well to such an ideal; most natural states or conditions describe continua between arbitrarily defined extremes. Human concepts are usually commensurately vague, and "where there is vagueness, there looms the sorites" (Hogan 1994 162). A practical corollary of the sorites paradox is that there is no point in looking for sharp boundaries where these are, by definition, nonexistent (Hyde 2011). To use common parlance, we should not attempt to "draw the line" as there is no clear "line" to be drawn.

\section{Life as a Soritean Phenomenon}

Does the sorites paradox apply to the distinction between "living" and "nonliving"? In the days when the vital force 
concept ruled, the answer to that question was firmly in the negative, as the distinction resulted from the presence or absence of a discrete entity, albeit a nebulous one. The difference between the two conditions was seen as a qualitative rather than a quantitative one. With the abandonment of vitalism, "life" came to be described more in process terms, i.e., what life does rather than what life is. The problem with this approach is that there does not appear to be any characteristic intrinsic and unique to life (Schulze-Makuch and Irwin 2008). A growing seed crystal in a school lab exhibits some of the characteristics of life: it grows, and moreover does so by absorbing and assimilating substances from its environment in such a way that there is a decrease in entropy-a sophisticated aspect of life. There are also the viruses to challenge any succinct definition of life in that they so clearly invoke a gray zone between the two states, exhibiting as they do so few "process" aspects of life. Even adding the capacity for evolution to the list of life attributes does not necessarily solve the problem as in a very simple state there may be reproduction without replication, thereby nullifying any Darwinian mechanism (Cleland and Chyba 2002 but cf. Bobrik et al. 2008 who make a theoretical case for Darwinian evolution at the molecular level).

Intelligent design theory may be regarded as a resurgence of the vitalist mindset in that it too posits a sharp line of demarcation between living and nonliving via its "irreducible complexity" clause. But life is a vague predicate and so no line of demarcation can be said to exist. Between a crystal and a eucaryotic cell, there exists a plethora of levels of organization; how we define life is likely to remain "no more than a matter of linguistic choice" (Cleland and Chyba 2002, 391). Life would appear to be a perfect candidate for the sorites paradox.

While hair-splitting over the definition of life is not particularly consequential when dealing with living entities that we know well in the world around us, developments in abiogenesis research brought a fresh impetus to bear on the issue as "the need became apparent for a definition that distinguishes between living and nonliving states, since that boundary (at least in concept) had to be crossed at some point in the past" (Schulze-Makuch and Irwin 2008, 12). In the heady early days of nineteenth century abiogenesis, the line was thought to have been crossed almost instantaneously when a given level of biochemical complexity was attained through simple organic substances organizing themselves into a cellular structure (bearing in mind that cell ultrastructure had hardly been elucidated at that time). Thus, when Haeckel "saw" the "organism" Bathybius haeckelii, there was no great surprise involved, as he had hypothesized that new life forms were constantly being spontaneously generated in seabed oozes; enigmatically, he saw no fundamental difference between living and nonliving anyway (Rehbock 1975; note also the hint of ongoing abiogenesis in Wächterschäuser 1988). We have come a long way since then. Drawing on the ideas of Oparin, Miller (1953) put abiogenesis on an experimental footing with his seminal laboratory work in which five amino acids were produced from a mixture of primordial gases, a line of inquiry which soon led to more refined experimental designs and commensurately more complex organic compounds. Theoreticians have increasingly tended to focus more on the evolution of biochemical processes than on the genesis of biochemical molecules (e.g., Fox 1981; Fleischaker 1990; Wächterschäuser 1988). Abiogenesis models now focus on a protracted, incremental transition from simple chemical systems with low levels of organization to cellular structures via systems of gradually increasing complexity and autonomy (see e.g., Ruiz-Mirazo et al. 2004; Schulze-Makuch and Irwin 2008). Terms such as "prebiotic," "protocells" and "precursor organisms" (Wächterschäuser 1988) abound in the abiogenesis literature, mostly in connection with organic entities and processes which already exhibit some characteristics of life. To quote Schulze-Makuch and Irwin (2008 15), "There was probably no sharp line but rather a gradual transition between a living and a non-living state of matter at the origin of life."

\section{Implications for Science Communicators and Educators}

Evolutionary biology is no stranger to soritean reasoning. In the evolutionary context, any taxonomic predicate is necessarily vague. To use the hackneyed ape-to-human transition as an example, the definition of both "ape" and "human" are arbitrary ones with ill-defined boundaries. The demarcation may, however, appear to be a clear one to an observer ignoring the temporal sequence between the two, which exists only in the remote past and is accordingly not visible, at least not to the untrained eye. The tendency of paleontologist to assign species names to every fossil find actually reinforces the illusion of clear boundaries as the posited sequence then implies "quantum leaps" from one discrete entity to another. Paleontologist, themselves recognize perfectly well that their "species" do not necessarily correspond to the standard classroom definition of a species, but most lay people - and perhaps some teachers - do not appear to be aware of the distinction.

The main focus of this article is, however, the application of the sorites paradox to life itself. For many people, the origin of life remains a major stumbling block to the acceptance of the evolutionary paradigm (Rice et al. 2010). Biological and chemical evolution are conflated in the public mindset, a situation at least in part attributable to the failure of much science education to come to grips with the distinction between the two (Vlaardingerbroek 2010). As long as life remains viewed as a discrete state, intuitive logic will continue 
to demand both a sharp line of demarcation between it and nonlife, and a definitive point of transition from one to the other locatable in precise time. The expression "the origin of life" in itself becomes problematic as it implies a definable "crossing of the line." Statements appearing in authoritative popular scientific literature-in this instance, Scientific American - such as, "It is virtually impossible to imagine how a cell's machines, which are mostly protein-based catalysts called enzymes, could have formed spontaneously as life first arose from non-living matter around 3.7 billion years ago" (Ricardo and Szostak 2009) exacerbate the situation. While unintended, the message conveyed by the second part of this sentence feeds into common misconceptions concerning both the arbitrary nature of life and the sudden, dramatic appearance thereof at a discrete point in time. The same may be said for textbook, classroom, and science media expressions such as "the first living cells."

It may seem semantically pedantic, but given that concepts and their concomitant "definitions" are by their very nature imprecise (Cleland and Chyba 2002; Hyde 2011); science communicators and educators need to revisit their phraseology with a view to avoiding the use of clichés which imply clear-cut boundaries where there are none. With regard to the origin of life, the geologic time scale throws us a lifeline: to claim that life "appeared" circa 3.7 billion years ago is hardly committing oneself to a discrete moment of transformation. It merely connotes that there were probably no biochemical systems to which the soubriquet living would be applied 3.8 billion years ago while, 200 million years later, there were in existence structures to which the term could be arbitrarily applied. The line that was crossed is a fuzzy one, at least in temporal terms, even if the timescale for abiogenesis is reduced to millennia rather than millions of years (as claimed by Emeline et al. 2003).

The transition from Euclidean reasoning to soritean reasoning is part-perhaps a defining feature - of intellectual maturation, certainly in the context of an understanding of modern biology. At lower levels of schooling, it is permissible to speak of what makes one thing living and another not. By the later stages of secondary schooling, and certainly at the tertiary stage, it is no longer so, and students need to be made aware of the indeterminateness of the concept of life. The classroom treatment of abiogenesis presents an excellent opportunity to do just that.

\section{References}

Bobrik M, Kvasnicka V, Pospichal J. Artificial chemical and molecular Darwinian evolution of DNA/RNA-like systems II - programmable folding. Stud Comput Intell. 2008;85:337-73.

Cleland CE, Chyba CF. Defining 'life'. Orig Life Evol Biosph. 2002;32:387-93.

Emeline AV, Otroshchenko VA, Ryabchuk VK, Serpone N. Abiogenesis and photostimulated heterogeneous reactions in interstellar media and on primitive Earth: relevance to the genesis of life. J Photochem Photobiol. 2003;3:203-24.

Fleischaker G. Origins of life: an operational definition. Orig Life Evol Biosph. 1990;20:127-37.

Fox SW. Origins of the protein synthesis cycle. Int J Quantum Chem. 1981;20:441-54.

Hogan T. Robust vagueness and the forced-march sorites paradox. Philos Perspect. 1994;8:159-88.

Hyde D. The sorites paradox. In: Ronzitti G, editor. Vagueness: a guide (logic, epistemology, and the unity of science). Berlin: Springer; 2011. p. 1-17. 19. http://www.springerlink.com/content/ x32776276h035r32/.

Miller SL. A production of amino acids under possible primitive earth conditions. Science. 1953;117:528-9.

Rehbock PF. Huxley, Haeckel, and the oceanographers: the case of Bathybius haeckelii. Isis. 1975;66:504-33.

Ricardo A, Szostak JW. The origin of life on Earth. Scientific American, August 2009. http://www.scientificamerican.com/article. cfm?id=origin-of-life-on-earth. Accessed 8 Jul 2011.

Rice JW, Warner DA, Kelly CD, Clough MP, Colbert JT. The theory of evolution is not an explanation for the origin of life. Evol Educ Outreach. 2010;3:141-2.

Ruiz-Mirazo K, Peretó J, Moreno A. A universal definition of life: autonomy and open-ended evolution. Orig Life Evol Biosph. 2004;34:323-46.

Schulze-Makuch D, Irwin LN. Definition of life. Life in the universe: advances in astrobiology and biogeophysics. 2008;3:7-24. http:// www.springerlink.com/content/28t826180k1j0147/

Vlaardingerbroek B. Abiogenesis in upper secondary biology curricula. Evol Educ Outreach. 2010;3:432-5.

Wächterschäuser G. Before enzymes and templates: theory of surface metabolism. Microbiol Rev. 1988;52:452-84. 